Journal of Mathematics and Statistics 8 (1): 82-84, 2012

ISSN 1549-3644

(C) 2012 Science Publications

\title{
A Kind of Intersection Graphs on Ideals of a Ring
}

\author{
Talebi, A.A. \\ Department of Mathematics, University of Mazandaran Babolsar, Iran
}

\begin{abstract}
Problem statement: Let $R$ be a ring. The graph $G(R)$ is the graph whose vertices are nontrivial ideal of $\mathrm{R}$ and in which two vertices $\mathrm{u}, \mathrm{v}$ are joined by an edge, if and only if $\mathrm{u} \cap \mathrm{v} \#\{0\}$. Approach: In this study we study some properties of $G(R)$. Results: We obtain conditions of $R$ such that $\mathrm{G}(\mathrm{R})$ is a path and determine the graph $\mathrm{G}(\mathrm{R})$ in which it is a tree. Conclusion: We conclude that ideals of $\mathrm{R}$ have degree one.
\end{abstract}

Key words: Graphs related, intersection graph, integers modulo, algebraic structure, distinct vertices, intersection graphs, obtain conditions

\section{INTRODUCTION}

Let $A=\left\{S_{i}: i \in I\right\}$ be an arbitrary family of sets. The intersection graph $G$ (A) is the graph whose vertices are $S_{i}, i \in I$ and in which the vertices $S_{i}, S_{j}$ are adjacent if and only if $S_{i} \neq S_{j}$ and $S_{i} \cap S_{j} \neq \phi$. It is more interesting to study the intersection graphs $\mathrm{G}(\mathrm{A})$ when the elements of A have an algebraic structure. These terdisciplinary studies allow us to obtain characterization and representation of especial classes of algebraic structure in terms of graphs and vice versa.

Many authors studied such graphs related to the group structure, for example Shen (2010) and Zelinka (1975). Various construction of graphs relative to the ring structure are found in Simis et al. (1994). Chakrabarty et al. (2009) studied the intersection graphs of ideal of rings. They determined the values of $n$ for which $G\left(Z_{n}\right)$ for ring $Z_{n}$ of integers modulo $n$ for $\mathrm{n} \in \mathrm{N}$ is connected, complete, bipartite, Eulerian and Hamiltonian.

For a given graph $\mathrm{H}$, the degree of a vertex $\mathrm{V}$ in $\mathrm{H}$ denoted by deg (v), is the number of edges incident to $\mathrm{v}$. A path $\mathrm{P}$ is a sequence of distinct vertices $\mathrm{v}_{1}, \mathrm{v}_{2}$, $\ldots v_{\mathrm{m}+1}$ in which very two consecutive vertices are adjacent. The number $\mathrm{m}$ is called the length of P. For two vertices $\mathrm{u}$ and $\mathrm{V}$ in a graph $\mathrm{H}$ the distance between $\mathrm{u}$ and $\mathrm{v}$, denoted by $\mathrm{d}(\mathrm{u}, \mathrm{v})$, is the length of the shortest path between $u$ and $v$, if such a path exist; otherwise we define $\mathrm{d}(\mathrm{u}, \mathrm{v})=\infty$.

A graph $\mathrm{H}$ is connected if there is a path between each pair of the vertices of $\mathrm{H}$ The diameter diam $(\mathrm{H})$ of a connected graph $\mathrm{H}$ is the maximum of $\mathrm{d}(\mathrm{u}, \mathrm{v})$ overall vertices $\mathrm{u}, \mathrm{v}$ The path $\mathrm{v}_{1}, \mathrm{v}_{2}, \ldots \mathrm{v}_{\mathrm{m}+1}$ is called a cycle if $\mathrm{v}_{1}=\mathrm{v}_{\mathrm{m}+1}$ A graph $\mathrm{H}$ is a tree if it be connected and have no cycle. A graph $\mathrm{H}$ with vertex set Eq. 1:

$\left\{\mathrm{u}, \mathrm{v}_{1}, \ldots, \mathrm{v}_{\mathrm{m}}\right\}$

And edge set $\mathrm{E}=\left\{\left\{\mathrm{u}, \mathrm{v}_{\mathrm{i}}\right\}: 1 \leq \mathrm{i} \leq \mathrm{m}\right.$ is called a star graph.

In this study, we determine conditions on ring $\mathrm{R}$ such that $G(R)$ is a tree or a path and get ideals of $R$ who's these degree is one.

\section{MATERIALS AND METHODS}

Consider the ring $Z_{n}$ of integers modulo $n$ for $n \in N$. We know that $Z_{n}$ is a principle ideal ring and each of these ideals is generated by $m \in Z_{n}$ where $m$ is a factor of $n$. Let $M$ denoted the set of all positive integers greater than one and which are not primes. In the following we have:

Theorem 1: [1] Let $n \in M$. The graph $G\left(Z_{n}\right)$ is complete if and only if $n=p^{m}$ where $P$ is a prime number and $\mathrm{m} \in \mathrm{N}, \mathrm{m}>1$.

We investigate the diameter $G\left(Z_{n}\right)$.

Obviously we have the following theorem:

- Theorem 2.2. For any $n \in M$, diam $\left(G\left(Z_{n}\right)\right) \leq 2$

- So clearly by Theorem 2.1 we have the following corollary

- Corollary 2.3. Let $n \in M$, then $\operatorname{diam}\left(\mathrm{G}\left(\mathrm{Z}_{\mathrm{n}}\right)\right)=2 \mathrm{if}$ and only if $n \neq p^{m}$

Corresponding Author: Talebi, A.A., Department of Mathematics, University of Mazandaran Babolsar, Iran 


\section{RESULTS}

We state the essential results of this study.

Proposition 3.1: Let I be a minimal ideal of a ring R. Let graph $\mathrm{G}(\mathrm{R})$ have no cycle of length 3 . Then we have $\operatorname{deg}(\mathrm{I})=1$, in $\mathrm{G}(\mathrm{R})$.

Proof: Let I be a minimal ideal of a ring $\mathrm{R}$ and $\operatorname{deg}(\mathrm{I})$ $\geq 2$. Let $J$ and $K$ are two distinct vertices of $G(R)$ such that both $\mathrm{J}$ and $\mathrm{K}$ are adjacent with vertex I Then $\mathrm{I} \cap \mathrm{J} \neq$ (0) and $\mathrm{I} \cap \mathrm{K} \neq(0)$. Thus $\mathrm{I} \cap \mathrm{J}=\mathrm{I}$, because $\mathrm{I} \cap \mathrm{J} \subseteq \mathrm{I}$ and $\mathrm{I}$ is minimal. Hence $\mathrm{I} \subseteq \mathrm{J}$. Similarly we get $\mathrm{I} \subseteq \mathrm{k}$. Thus $\mathrm{I} \subseteq \mathrm{J} \cap \mathrm{k}$ and this implies that $\mathrm{J}$ and $\mathrm{K}$ are adjacent. Thus the sequence $I, J, K$ of vertices of $G(R)$ is a cycle of length 3 , a contradiction.

Theorem 3.2: For ring $G(R) \approx P_{2}$, if and only if $R$ have only two ideal, namely minimal ideal and maximal ideal.

Proof: Let $\mathrm{G}(\mathrm{R}) \approx \mathrm{P}_{2}$ and $\mathrm{J}, \mathrm{K}$ are two vertices of $\mathrm{G}(\mathrm{R})$ Then $I \cap J=I$ or $I \cap J=J$. If $I \cap J=I$, then $I \subseteq J$. This implies that $\mathrm{I}$ is minimal ideal and $\mathbf{J}$ is maximal ideal. If $\mathrm{I} \cap \mathrm{J}=\mathrm{J}$, similarly we conclude that $\mathrm{J}$ is minimal ideal and I is maximal ideal. Conversely, for nontrivial graph $G(R)$ is obvious.

Example: For ring $\mathrm{R}=\mathrm{Z}_{\mathrm{p} 3}$, there is only two nontrivial ideal $\mathrm{I}=(\mathrm{a}), \mathrm{J}=\left(\mathrm{a}^{2}\right)$ and we have $\mathrm{G}(\mathrm{R}) \approx \mathrm{P}_{2}$.

Lemma 3.3: Let $I$ be a vertex of $G(R)$ such that deg $(I)=1$. Then $I$ is a minimal ideal or maximal ideal.

Proof: Let for vertex I, deg (I)=1 and J be only vertex of $G(R)$ such that $J$ is adjacent to $I$. Then by definition, $I \cap J \neq(0)$. Since $\operatorname{deg}(I)=1, I \cap J=I$ or,$I \cap J=J$. Thus $\mathrm{I} \subseteq \mathrm{J}$ orJ $\subseteq \mathrm{I}$. If $\mathrm{I} \subseteq \mathrm{J}$, then there is not nontrivial ideal $\mathrm{L}$ such that $\mathrm{L} \subset \mathrm{I}$, because, deg $(\mathrm{I})=1$. Hence $\mathrm{I}$ is minimal ideal. If $\mathrm{J} \subset \mathrm{I}$, then there is not ideal $\mathrm{L}$ such that $\mathrm{I} \subset \mathrm{L} \subset$ $R$, because $\operatorname{deg}(I)=1$. Thus I is maximal.

Lemma 3.4: Let $G(R)$ is a path as sequence $I_{1}, I_{2}, . . I_{t}$. If $I_{1}$ be a maximal ideal of $R$, then $G(R) \approx P_{2}$.

Proof: Let $I_{1}$ be maximal ideal. We know that $I_{1} \cap I_{2}$ $=I_{1}$ or vertices $I_{1} \cap I_{2}$ and $I_{1}$ are adjacent. If $I_{1} \cap I_{2}=I_{1}$, then $I_{2}=I_{1}$, because $I_{1} \subseteq I_{2}$ and $I_{1}$ is maximal. This is a contradiction with $I_{2} \neq I_{1}$. Thus $I_{1} \cap I_{2}=I_{2}$ and so $I_{2} \subseteq I_{1}$. Let $\mathrm{n} \geq 3$. Therefore $(0) \neq \mathrm{I}_{2} \cap \mathrm{I}_{3}=\mathrm{I}_{2}$ or vertices $\mathrm{I}_{2} \cap \mathrm{I}_{3}$ and $1_{2}$ are adjacent. If $(0) \neq I_{2} \cap I_{3}=I_{2}$, then $I_{2} \subseteq I_{3}$ and so $I_{2} \subseteq I_{3} \cap I_{1}$. Thus vertices $I_{1}$ and $I_{3}$ are adjacent, a contradiction. Otherwise we have $\mathrm{I}_{2} \cap \mathrm{I}_{3}=\mathrm{I}_{1}$ or $\mathrm{I}_{2} \cap \mathrm{I}_{3}=\mathrm{I}_{3}$. If $\mathrm{I}_{2} \cap \mathrm{I}_{3}=\mathrm{I}_{3}$, then $\mathrm{I}_{1}$ and $\mathrm{I}_{3}$ are adjacent, a contradiction. But if $\mathrm{I}_{2} \cap \mathrm{I}_{3}=\mathrm{I}_{3}$, then $\mathrm{I}_{3} \subseteq \mathrm{I}_{2}$. Now in view of $\mathrm{I}_{2} \subseteq \mathrm{I}_{1}$ we get that vertices $I_{1}$ and $I_{3}$ are adjacent, a contradiction.

Theorem 3.5: Let $G(R)$ is a path, then $G(R) \cong P_{2}$ or $\mathrm{G}(\mathrm{R}) \approx \mathrm{P}_{3}$. If $\mathrm{G}(\mathrm{R}) \approx \mathrm{P}_{3}$, then $\mathrm{R}$ have only three ideals $\mathrm{I}_{1}, \mathrm{I}_{2}, \mathrm{I}_{3}$ such that $\mathrm{I}_{2}=\mathrm{I}_{1} \oplus \mathrm{I}_{3}$.

Proof: Let $G(R)$ be a path as sequence $I_{1}, I_{2}, \ldots I_{n}$. By Lemma 3.3, the ideal $\mathrm{I}_{1}$ is a minimal ideal or maximal ideal. Let $\mathrm{I}_{1}$ be a minimal ideal. We have $\mathrm{I}_{1} \cap \mathrm{I}_{2} \neq(0)$, because vertices $I_{1}$ and $I_{2}$ are adjacent. Thus $I_{2} \cap I_{3}=I_{1}$, because $I_{1} \cap I_{2} \subseteq I_{1}$ and $I_{1}$ is a minimal ideal and hence $\mathrm{I}_{1} \subseteq \mathrm{I}_{2}$. Let $\mathrm{n}>2$, then $\mathrm{I}_{2} \cap \mathrm{I}_{3 \neq} \mathrm{I}_{1}$, because $\mathrm{I}_{2} \cap \mathrm{I}_{3}=\mathrm{I}_{2}$ implies $\mathrm{I}_{2} \subseteq \mathrm{I}_{3}$ and in view of $\mathrm{I}_{1} \subseteq \mathrm{I}_{2}$, we conclude that $\mathrm{I}_{1}$ and $\mathrm{I}_{3}$ are adjacent, that is a contradiction. Hence $I_{2} \cap I_{3}=I_{1}$ or $I_{2} \cap I_{3}=I_{3}$. If $I_{2} \cap I_{3}=I_{1}$, then $I_{1}$ and $I_{3}$ are adjacent, a contradiction. Thus $I_{2} \cap I_{3}=I_{1}$ and so $I_{3} \subseteq I_{2}$. Thus, this is true for $n=3$. Let $n>3$. If $I_{4} \cap I_{3}=I_{3}$, in view of $I_{3} \subseteq I_{2}$ we conclude that $\mathrm{I}_{3}$ and $\mathrm{I}_{4}$ are adjacent, a contradiction. Thus vertices $I_{3} \cap I_{4}$ and $I_{3}$ are adjacent. So, $I_{4} \cap I_{3}=I_{2}$ or $\mathrm{I}_{4} \cap \mathrm{I}_{3}=\mathrm{I}_{4}$. If $\mathrm{I}_{4} \cap \mathrm{I}_{3}=\mathrm{I}_{4}$, then $\mathrm{I}_{4} \subseteq \mathrm{I}_{3}$ and so $\mathrm{I}_{4} \subseteq \mathrm{I}_{3} \subseteq \mathrm{I}_{2}$. This implies that $\mathrm{I}_{2}$ and $\mathrm{I}_{4}$ are adjacent, a contradiction. If $\mathrm{I}_{4} \cap \mathrm{I}_{3}=\mathrm{I}_{2}$, then $\mathrm{I}_{1} \subseteq \mathrm{I}_{2} \subseteq \mathrm{I}_{3}$. Thus $\mathrm{I}_{1}$ and $\mathrm{I}_{3}$ are adjacent, a contradiction. In final, by conditions $I_{1} \subseteq I_{2}, I_{3} \subseteq I_{2}$ and this fact that $\mathrm{I}_{1}$ and $\mathrm{I}_{3}$ are not adjacent we conclude $\mathrm{I}_{2}=\mathrm{I}_{1}$ $\oplus \mathrm{I}_{3}$.

If $I_{1}$ be maximal ideal we conclude the theorem by Lemma 3.4.

By a similar argument as in the proof of Theorem 3.5 we see that in a tree, length of a path with end points of degree 1 is at most 2 . Thus we obtain the following Corollary:

Corollary 3.6: For a ring $R$, the graph $G(R)$ is a tree if and only if is a star graph.

\section{DISCUSSION}

We studied a especial class of a algebraic structure (the ring structure) in terms of graphs and vice versa. Using the properties of ideals in a ring, we discussed some of the properties of the graph $G(R)$.

\section{CONCULSION}

Using notions graph theory and group theory we characterized the graph $G(R)$, when it is a path or 
tree, determined ideals $I$, when $\operatorname{deg}(I)=1$ in the graph $G(R)$. Also we obtained a bound for $\operatorname{diam}\left(G\left(Z_{n}\right)\right)$.

\section{ACKNOWLEDGEMENT}

The researcher would like to thank to the Research centre of algebra hyper structures and Fuzzy Mathematics, Bobolsar, Iran for their supports.

\section{REFERENCES}

Chakrabarty, I., S. Ghosh, T.K. Mukherjee and M.K. Sen, 2009. Intersection graphs of ideals of rings. Discrete Math., 309: 5381-5392 DOI: 10.1016/j.disc.2008.11.034
Shen, R., 2010. Intersection graphs of subgroups of finite groups. Czechoslovak Math J., 60: 945-950. DOI: 10.1007/s10587-010-0085-4

Simis, A. W.V. Vasconcelos and R.H. Villarreal, 1994. On the ideal theory of graphs. J. Algebra, 167: 389416. DOI: 10.1006/jabr.1994.1192

Zelinka, B., 1975. Intersection graphs of finite abelian groups. Czechoslovak Math J., 25: 171-174. 